\section{Contouring by electronic speckle pattern interferometry with quadruple-beam illumination}

\section{Y. Zou, H. Diao, X. Peng, and H. Tiziani}

Institute of Applied Optics, University of Stuttgart, Pfaffenwaldring 9, 7000 Stuttgart 80, Germany. Received 16 October 1991. 0003-6935/92/316599-04\$05.00/0.

(C) 1992 Optical Society of America.

We present a new arrangement for contouring by electronic speckle pattern interferometry with four illumination beams, thereby making it unnecessary to move anything during the measurement.

Key words: Contouring, interferometry, speckle phenomena, metrology

Optical contouring techniques applied on a rough surface by means of electronic speckle pattern interferometry (ESPI) have been developed for three-dimensional shape analysis and topography measurement. ${ }^{1-5}$ Correlation fringes are shown after the subtraction or addition of the video signals from two speckle patterns. One can obtain the contour data from the correlation fringes by using a phase-shifting method and algorithm. ${ }^{6}$ The existing contouring methods by ESPI consist of using two-wavelength illumination $^{1}$ or altering the object illumination beam ${ }^{2,3}$ in a conventional ESPI system with a smooth interference reference beam and altering the object ${ }^{4}$ or shifting the illumination beams ${ }^{5}$ in a dual-beam system.

We present an inexpensive ESPI arrangement where nothing is moved to obtain contour fringes. Once this system is well adjusted, it can be used simply and practically, e.g., in a black box with a complete program package for fringe analysis. The schematic of such an arrangement is shown in Fig. 1. The collimated laser beam is split by the first beam splitter $\mathrm{BS}_{1}$, then combined again with the second prism beam splitter $\mathrm{BS}_{2}$ with a small angle difference. Mirror $\mathrm{M}_{5}$ plays the role of a direction compensator for forming correct contour planes. ${ }^{5}$ The first speckle pattern is taken by the CCD camera when shutter $S_{1}$ is open and shutter $S_{2}$ is closed and then subtracted from the second 
speckle pattern when $S_{1}$ is closed and $S_{2}$ is open. The resulting correlation fringes are displayed on a TV monitor, and the fringe video signals are taken and processed by the computer incorporating an image frame grabber board for fringe analysis when the phase-shifting method is used.

The geometry for forming the correlation fringes is similar to that in Ref. 7. The intensity difference at viewpoint $\mathrm{P}$ (see Fig. 2) between these two speckle patterns is

$$
\begin{aligned}
\Delta \Gamma= & 4\left(I_{1} I_{2}\right)^{1 / 2} \sin \left[\left(\phi_{s 1}-\phi_{s 2}\right)+\left(\Psi_{m}+\Psi_{m}{ }^{\prime}\right) / 2\right] \\
& \times \sin \left(\Delta \Psi_{m} / 2\right),
\end{aligned}
$$

where $I_{1}$ and $I_{2}$ are the intensities of two illumination beams scattered from point $M$ in the object for one speckle pattern, $\phi_{s 1}$ and $\phi_{s 2}$ are the random phases of these two beams, $\Psi_{m}$ and $\Psi_{m}{ }^{\prime}$ are the phase differences between these two beams caused by the object shape for the two speckle patterns, respectively, and $\Delta \Psi_{m}=\Psi_{m}{ }^{\prime}-\Psi_{m} . I_{1}, I_{2}, \phi_{s 1}$, and $\phi_{s 2}$ are assumed to have undergone no change in the two speckle patterns. ${ }^{1}$ The sin term with the random phases $\phi_{s 1}$ and $\phi_{s 2}$ in Eq. (1) disappears after averaging and high-pass filtering while the signal is displayed on the monitor. Hence the correlation fringes depend on only the phase term $\Delta \Psi_{m} . \quad \Delta \Psi_{m}$ can be determined from the discussion below.

In Fig. $2 \mathbf{K}_{1}$ and $\mathbf{K}_{2}$ are the unit direction vectors of the two illumination beams during the first exposure, $\mathbf{K}_{1}{ }^{\prime}$ and $\mathbf{K}_{2}{ }^{\prime}$ are those at the second exposure, $\Delta \theta$ is the angle difference between the illumination beams for the two speckle patterns, $\theta$ is the illumination angle of both illumination beams if they are set at the same angle with respect to the view direction $Z, \mathbf{r}_{m}$ is the position vector from point $\mathrm{P}$ to point $\mathrm{M}, \beta$ is the angle between $\mathbf{r}_{m}$ and the $Z$ axis. If we ignore the constant optical paths of the two illumination beams from the light source to viewpoint $P$, the phase advances of the two illumination beams scattered from $M$ to $P$ for the first speckle pattern are

$$
\begin{aligned}
& \phi_{m 1}=\frac{2 \pi}{\lambda}\left(r_{m}+\mathbf{r}_{m} \cdot \mathbf{K}_{1}\right), \\
& \phi_{m 2}=\frac{2 \pi}{\lambda}\left(r_{m}+\mathbf{r}_{m} \cdot \mathbf{K}_{2}\right),
\end{aligned}
$$

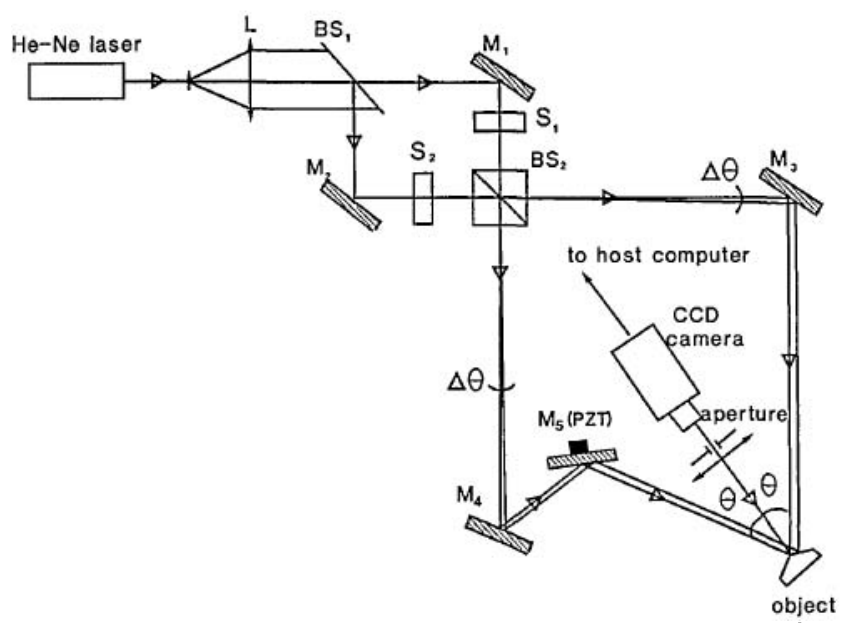

Fig. 1. Schematic of the ESPI arrangement with quadruple-beam illumination. L, collimator lens.

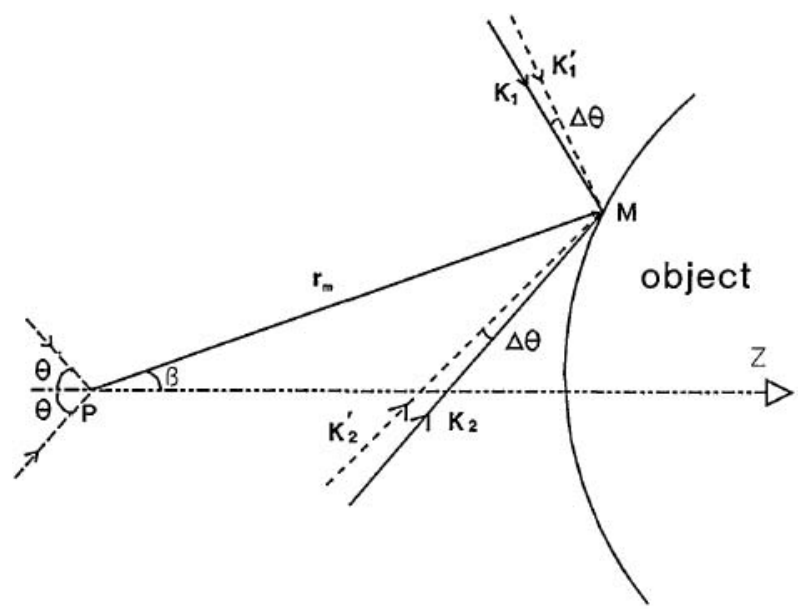

Fig. 2. Geometry of the ESPI contouring.

respectively, where $\lambda$ is the wavelength of the illuminating light and $r_{m}$ is the scalar magnitude of $\mathbf{r}_{m}$. Therefore

$$
\begin{aligned}
\Psi_{m} & =\phi_{m 1}-\phi_{m 2} \\
& =\frac{2 \pi}{\lambda} \mathbf{r}_{m} \cdot\left(\mathbf{K}_{1}-\mathbf{K}_{2}\right) .
\end{aligned}
$$

Similarly, for the second speckle pattern we have

$$
\Psi_{m}{ }^{\prime}=\frac{2 \pi}{\lambda} \mathbf{r}_{m} \cdot\left(\mathbf{K}_{1}^{\prime}-\mathbf{K}_{2}^{\prime}\right)
$$

Then

$$
\begin{aligned}
\Delta \Psi_{m} & =\frac{2 \pi}{\lambda} \mathbf{r}_{m} \cdot\left(\Delta \mathbf{K}_{1}-\Delta \mathbf{K}_{2}\right) \\
& =\frac{2 \pi}{\lambda}\left|\mathbf{r}_{m}\right|\left|\Delta \mathbf{K}_{1}-\Delta \mathbf{K}_{2}\right| \cos \beta,
\end{aligned}
$$

where $\Delta \mathbf{K}_{1}=\mathbf{K}_{1}{ }^{\prime}-\mathbf{K}_{1}$ and $\Delta \mathbf{K}_{2}=\mathbf{K}_{2}{ }^{\prime}-\mathbf{K}_{2}$. It can be found from Fig. 2 that $\left|\Delta \mathbf{K}_{1}\right|=\left|\Delta \mathbf{K}_{2}\right|=\Delta \theta\left|\mathbf{K}_{1}\right|=\Delta \theta\left|\mathbf{K}_{2}\right|=$ $\Delta \theta$, where $\left|\mathbf{K}_{1}\right|=\left|\mathbf{K}_{2}\right|=1$ and from Fig. 3 that $\mid \Delta \mathbf{K}_{1}-$ $\Delta \mathbf{K}_{2}|=2 \sin \theta| \Delta \mathbf{K}_{1} \mid=2 \sin \theta \Delta \theta$. Finally we have

$$
\begin{aligned}
\Delta \Psi_{m} & =\frac{2 \pi}{\lambda}(2 \sin \theta) \Delta \theta h, \\
h & =r_{m} \cos \beta,
\end{aligned}
$$

where $h$ is the depth of the object along the view direction $Z$ when the angle changes of $\mathbf{K}_{1}$ and $\mathbf{K}_{2}$ are antisymmetric (see Fig. 2). Therefore the contour interval is as follows:

$$
d=\frac{\lambda}{(2 \sin \theta) \Delta \theta} .
$$

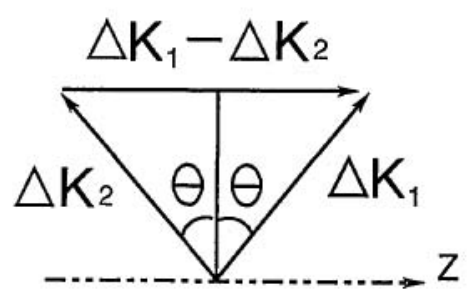

Fig. 3. Vector geometry. 


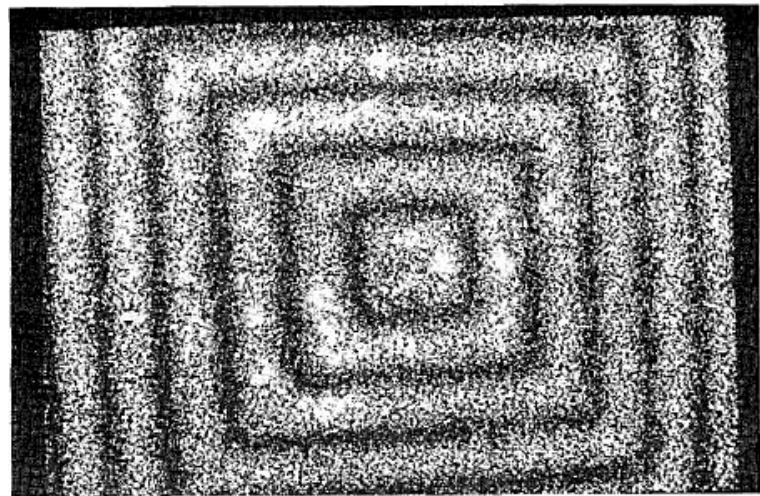

(a)

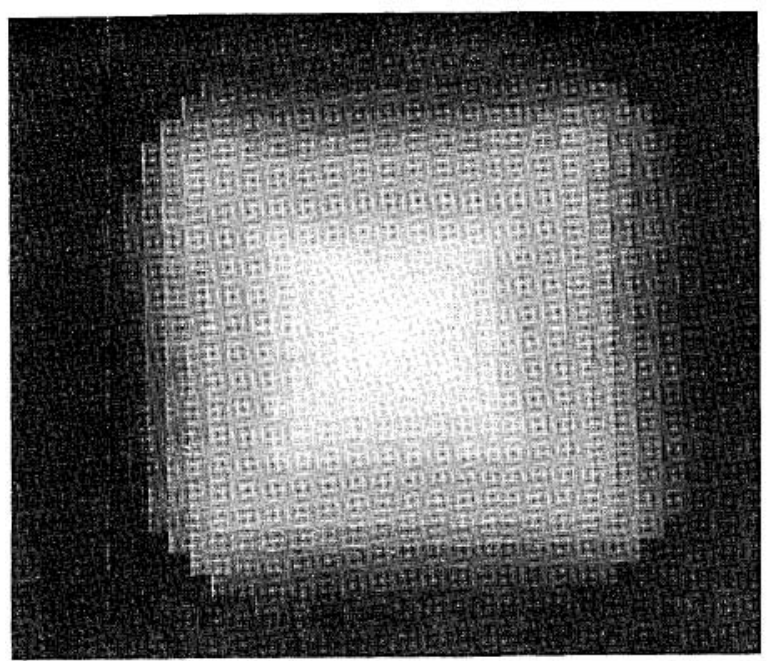

(b)

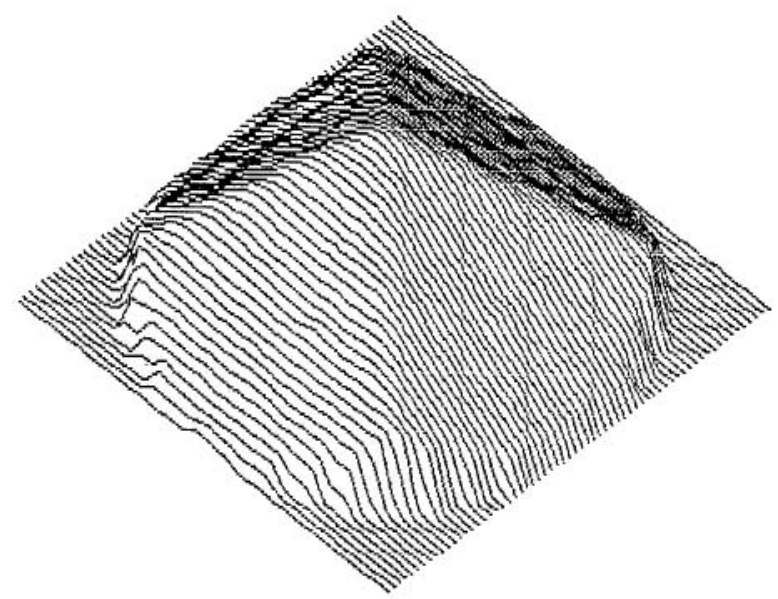

(c)

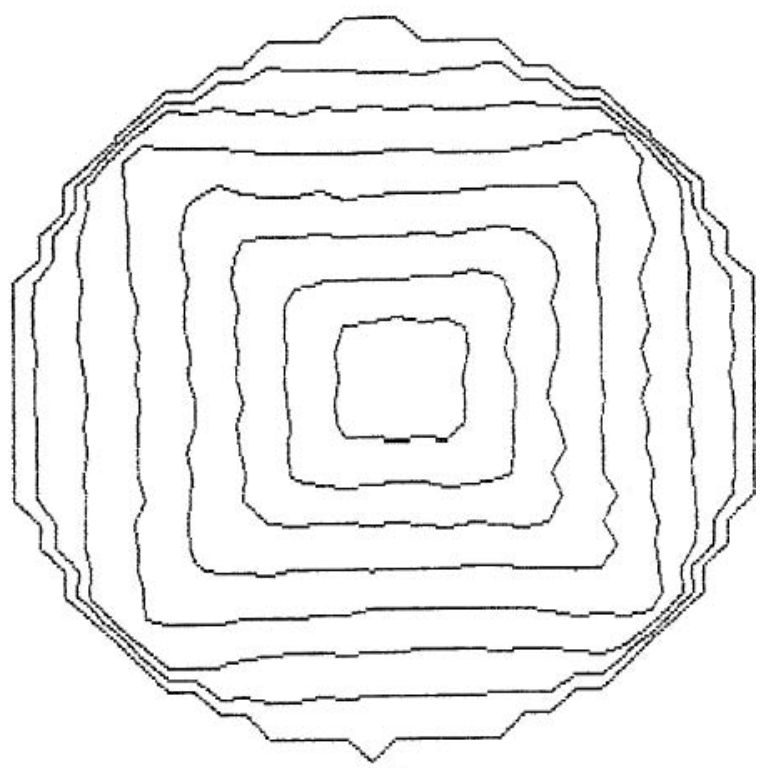

(d)

Fig. 4. Results obtained when the illumination angle $\theta$ is $30^{\circ}$ and the angle difference $\Delta \theta$ is $0.77 \mathrm{mrad}$ : (a) contour fringes, (b) phase map, (c) three-dimensional plot, (d) contour map.

The sensitivity can be controlled by altering the size of $\Delta \theta$ in our arrangement as we did in Fig. 1 by tilting mirror $\mathrm{M}_{1}$ or mirror $\mathrm{M}_{2}$.

A $10-\mathrm{mW}$ He-Ne laser was used as a coherent illumination source. The test object was a pyramid with an apex angle of $120^{\circ}$. The CCD camera was a Sony XC-77CE with a resolution of $11 \mu \mathrm{m}$. The illumination angle $\theta$ was $30^{\circ}$, and the angle difference $\Delta \theta$ was $0.77 \mathrm{mrad}$. Note that we used a cube (prism) beam splitter $\mathrm{BS}_{2}$ in the arrangement to simplify the adjustment of the four beams. The piezoelectric-transducer-(PZT)-driven mirror $\mathrm{M}_{5}$ introduced phase shifting during the second exposure for phase evaluation. The images were processed by an Epson host computer with a FG-100-AT frame grabber board. Three interferograms with a shifted phase of $\pi / 2$ with respect to one other were used for evaluating the phases. ${ }^{4}$ A $30 \times 29$ sampling array with a gray level of 10 was employed to digitize the interferograms; $3 \times 3$ and $5 \times 5$ convolution filtering was used sequentially to smooth the speckles in the interferograms. Figure 4(a) shows the contour fringes of the object surface. Figures 4(b), 4(c), and 4(d) show the phase map, the three-dimensional plot, and the contour map evaluated from the three phase-shifted intensity data, respectively.
A naturally supplemental result is shown by a similar arrangement with three illumination beams. One beam plays only the role of a reference wave to form the interference speckle patterns. The other two beams with small angle differences, which are the illumination waves for the first and second exposures, respectively, should be at incident angles of $\sim 90^{\circ}$ to the view direction ${ }^{7}$ so that contour fringes can be obtained.

The new ESPI arrangement presented above has obvious advantages. The setup is relatively stable during the process because there is no further mechanical movement after adjustment; thus fast automatic processing results. The sensitivity can be selected during the adjustment and corrected for further measurements.

The authors thank G. Padrini for helpful discussions. The financial support from the Alexander von Humboldt Foundation to X. Peng is gratefully acknowledged.

\section{References}

1. R. Jones and C. Wykes, Holographic and Speckle Interferometry (Cambridge U. Press, Cambridge, 1989), Chap. 5, p. 204.

2. B. D. Bergquist and P. Montgomery, "Contouring by electronic 
speckle pattern interferometry," in Optics in Engineering Measurement, W. F. Fagan, ed., Proc. Soc. Photo-Opt. Instrum. Eng. 599, 189-195 (1985).

3. S. Winther and G. Slettemoen, "An ESPI contouring technique in strain analysis," in Symposium Optika '84, G. Lupkovics and A. Podmaniczky, eds., Proc. Soc. Photo-Opt. Instrum. Eng. 473, 44-47 (1984).

4. C. Joenathan, B. Pfister, and H. Tiziani, "Contouring by electronic speckle pattern interferometry employing dual beam illumination," Appl. Opt. 29, 1905-1911 (1990).
5. X. Peng, H. Y. Diao, Y. L. Zou, and H. Tiziani, "Contouring by modified dual-beam ESPI based on tilting illumination beams," Optik (Stuttgart) 90, 61-64 (1992).

6. K. Creath, "Phase-shifting speckle interferometry," Appl. Opt. 24, 3053-3058 (1985).

7. Y. Zou, H. Diao, X. Peng, and H. Tiziani, "Geometry for contouring by electronic speckle pattern interferometry based on shifting illumination beams," Appl. Opt. 31, 6616-6621 (1 1992). 